\title{
Kufeld, Walter (Hrsg.) (2013): Klimawandel und Nutzung von regenerativen Energien als Herausforderungen für die Raumordnung
}

\author{
Hannover: Akademie für Raumforschung und Landesplanung. = Arbeitsbericht \\ der ARL 7. $265 \mathrm{~S}$.
}

\section{Thomas Zimmermann}

Online publiziert: 29. Mai 2014

(C) Springer-Verlag Berlin Heidelberg 2014

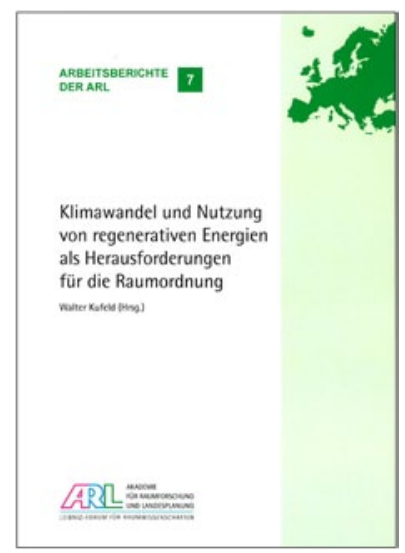

Seit der Veröffentlichung des vierten Berichts des „Intergovernmental Panel on Climate Change“ (IPCC) im Jahr 2007 gewinnt ein umfassender Umgang mit dem Klimawandel in der räumlichen Planung zunehmend an Bedeutung. Dabei können drei unterschiedliche Handlungsfelder voneinander abgegrenzt werden: die Förderung der Erzeugung von regenerativen Energien, die Senkung des Energieverbrauchs durch Suffizienz-, Einsparungs- und Effizienzstrategien sowie die Anpassung an die Folgen der klimatischen Veränderungen (Davoudi 2009: 18). Grundsätzlich handelt es sich dabei nicht um ein vollständig neues Handlungsfeld. Beispielsweise wurden in Deutschland die $\mathrm{CO}_{2}$-effizienten Raumstrukturen bereits Anfang der 1990er Jahre als planungsbezogene Handlungsmöglichkeiten zur Senkung des

T. Zimmermann ( $\square)$

Fachgebiet Stadtplanung und Regionalentwicklung, HafenCity Universität Hamburg,

Großer Grasbrook 9, 20457 Hamburg, Deutschland

E-Mail: thomas.zimmermann@hcu-hamburg.de
Energieverbrauchs thematisiert und diskutiert (Bergmann/ Kanzlerski/Otto et al. 1993). In der Folgezeit gewannen mit der Steuerung des Ausbaus der Windenergie Aspekte der Förderung von regenerativen Energien und mit dem vorbeugenden Hochwasserschutz das Themenfeld der Klimaanpassung an Bedeutung. In den vergangenen Jahren förderte der Bund die Erstellung von umfassenden raumplanerischen Klimaanpassungsstrategien in regionalen Modellvorhaben im Rahmen des KlimaMORO ${ }^{1}$. Der Ausbau des Einsatzes von erneuerbaren Energien und die Senkung des Energieverbrauchs stehen im Mittelpunkt des derzeit laufenden Modellvorhabens „Regionale Energiekonzepte als strategisches Instrument der Landes- und Regionalplanung“.

Der Arbeitsbericht der Akademie für Raumforschung und Landesplanung (ARL) „Klimawandel und Nutzung von regenerativen Energien als Herausforderungen für die Raumordnung" widmet sich dem Umgang mit dem Klimawandel in der räumlichen Planung am Beispiel Bayerns. Der eigene Anspruch besteht dabei darin, Leitbilder, Strategien und Instrumente im Kontext Klimawandel und Energiewende und damit raumplanerische Handlungsmöglichkeiten und zu bewältigende Herausforderungen zu thematisieren. Die Veröffentlichung bildet das Ergebnis der mehrjährigen Zusammenarbeit einer Arbeitsgruppe der Landesarbeitsgemeinschaft Bayern der ARL unter der Leitung von Walter Kufeld. Ihre Beiträge sind sowohl von Wissenschaftlern als auch von mit Fragen des Klimawandels und der Energiewende befassten Praktikern aus Bayern verfasst.

Die Einleitung gibt einen Überblick über die raumordnerischen Handlungsfelder beim Umgang mit dem Klimawandel in der räumlichen Planung und fasst die zentralen Botschaften der Arbeitsgruppe in den „Zugspitz-Thesen“ (vgl. auch ARL 2012) zusammen. Mit den beiden Bei-

${ }^{1}$ Vgl. http://klimamoro.de (07.02.2014). 
trägen des ersten Abschnittes verlässt der Sammelband zunächst die raumplanerische Perspektive und widmet sich den politischen Zielen Bayerns in diesem Politikfeld und den Ausbaupotenzialen der unterschiedlichen Träger von erneuerbaren Energien. Der zweite Abschnitt unterstreicht die raumordnerische Relevanz der Energie- und Klimaschutzziele. Der erste Beitrag thematisiert raumstrukturelle Aspekte von Photovoltaik und Biomasse. Im Anschluss stellt ein weiterer Beitrag die Klimarelevanz der Siedlungsentwicklung und damit eines klassischen raumordnerischen Handlungsfeldes heraus. Anhand von bayerischen Fallbeispielen beschreibt der dritte Abschnitt raumplanerische Instrumente und Koordinationsaufgaben. Darüber hinaus wird in dem Beitrag „Begleitumstände und Bestimmungsgründe einer problemangemessenen bayerischen Raumordnung“ die Rolle der Raumordnung bei der Transformation zu einer nachhaltigen Gesellschaft diskutiert. Die Beschreibung von Modellprojekten sowohl zur Klimaanpassung in den oberbayerischen Alpen und im Landkreis Neumarkt als auch zum verstärkten Einsatz erneuerbarer Energien in Form von Elektromobilität bildet den Schwerpunkt des vierten Abschnitts. Der abschließende Beitrag fasst die Erkenntnisse der Arbeitsgruppe zusammen, indem er zunächst die Raumrelevanz des Umgangs mit dem Klimawandel herausstellt und im Anschluss auf (partizipative) Instrumente eingeht.

Die thematisch breit angelegte Publikation ermöglicht punktuelle Einblicke in die gegenwärtig von der Raumordnung in Bayern bearbeiteten Handlungsfelder beim Umgang mit dem Klimawandel. Dabei stehen die zumeist praktischen Erfahrungen der Autoren im Vordergrund. Einher geht damit, dass die einzelnen Beiträge theoretische Bezüge weitestgehend ausblenden. Auch können aus den einzelnen Beiträgen keine landesweit generalisierbaren Aussagen zum Stand des Umgangs mit dem Klimawandel in der bayerischen Raumplanung getroffen werden. Dementsprechend erfolgt auch keine Einordnung in die bundesdeutsche Planungspraxis. Der thematische Schwerpunkt des Sammelbandes liegt auf dem gegenwärtig politisch aktuellen Thema Energiewende und dabei insbesondere auf dem Ausbau der erneuerbaren Energien. Die Bedeutung der Raumordnung für die ebenfalls erforderliche Energieeinspa- rung thematisiert der Sammelband mit der Klimarelevanz von Siedlungsstrukturen am Rande. Hier wäre eine intensivere Auseinandersetzung mit den bisher verfolgten Leitbildern und Steuerungsansätzen sowie ihren Wirkungen auf das erklärte Ziel der $\mathrm{CO}_{2}$-Einsparung wünschenswert gewesen. In Ansätzen behandelt ein Beitrag raumordnerische Handlungsansätze bei der Transformation in eine nachhaltige Gesellschaft. Daraus folgende strategische Handlungsfelder, wie die Hinwendung zu Suffizienzstrategien, thematisieren die Beiträge des Sammelbandes leider nicht. Dies erstaunt nicht, da entsprechende Überlegungen den wissenschaftlichen Diskurs bisher nicht verlassen haben. Die thematische Fokussierung verdeutlicht darüber hinaus, dass die Anpassung an die Folgen der klimatischen Veränderungen für die praktische Arbeit der Raumordnung bisher kaum relevant ist. Hier reichen die beschriebenen Erfahrungen bisher nicht über Modellprojekte hinaus. Dementsprechend stellt sich dem Lesenden die Frage, inwieweit die Raumordnung in ihrer alltäglichen Arbeit entsprechende Fragestellungen aufgreift. Insgesamt gibt der Sammelband damit den Stand des gegenwärtigen raumplanerischen Diskurses zum Umgang mit dem Klimawandel gut wieder. Zur Lektüre sei er all jenen empfohlen, die einen Überblick über unterschiedliche Aspekte der planungspraktischen Bewältigung von Herausforderungen des Klimawandels gewinnen wollen.

\section{Literatur}

ARL - Akademie für Raumforschung und Landesplanung (2012): „Zugspitz-Thesen“: Klimawandel, Energiewende und Raumordnung. Hannover. $=$ Positionspapier aus der ARL 90.

Bergmann, E.; Kanzlerski, D.; Otto, I.; Peters, A.; Schmitz, S.; Wagner, G.; Wiegandt, C.-C. (1993): Raumstruktur und $\mathrm{CO}_{2}$-Vermeidung. In: Informationen zur Raumentwicklung 8, 489-567.

Davoudi, S. (2009): Framing the role of spatial planning in climate change. o. O. = Global Urban Research Unit, Electronic Working Paper 43. http://www.ncl.ac.uk/guru/publications/working/documents/EWP43. $\operatorname{pdf}(02.05 .2014)$.

IPCC - Intergovernmental Panel on Climate Change (2007): Fourth Assessment Report of the Intergovernmental Panel on Climate Change. Genf. 